\title{
Calculus in a paraureteral diverticulum masquerading as calculus at the ureterovesical junction on unenhanced helical CT scan
}

\begin{abstract}
Unenhanced Helical CT scan done in a prone position differentiates a calculus at the ureterovesical junction from the a calculus in abladder lying near the ureteric orifice by the fact that the calculus at the ureterovesical junction doesnot change its position. However a calculus in a paraureteral diverticulum mimicas a calculus at the ureterovesical junction and maynot show change in position in prone scan if the diverticulum has a narrow mouth. We present a case to highlight this point.
\end{abstract}

Keywords: paraureteral diverticulum, calculus, unenhanced helical ct scan
Volume 3 Issue $6-2017$

\author{
Anshu Sharma,' Gyanendra Sharma ${ }^{2}$ \\ 'Chitale Clinic Pvt. Ltd, India \\ ${ }^{2}$ Department of Radiology, India
}

Correspondence: Gyanendra Sharma, Onkar Nilayam, 3/27 Samarth Nagar, North Sadar Bazar, Solapur-41300I, Maharashtra, India, Tel 919822195058,

Emaildrgrsharma@gmail.com

\section{Introduction}

Unenhanced Helical CT Scan is now an established modality to evaluate patients presenting with flank pain and has a sensitivity of $97 \%$ to diagnose ureteral calculi. ${ }^{1}$ As many as $18 \%$ of patients with acute flank pain and obstructing ureteral calculus have calculus located at the ureterovesical junction at the time of diagnosis. ${ }^{2}$ A calculus at the ureterovesical junction is distinguished from a vesical calculus lying near the ureteral orifice by performing the unenhanced helical scan in the prone position where the vesical calculus falls while the calculus at the ureterovesical junction does not fall. ${ }^{3}$ We present a case which was an exception to this rule and a vesical calculus in a paraureteral diverticulum masqueraded as an ureterovesical junction calculus on Unenhanced helical scan done both in supine and prone position.

\section{Case presentation}

A 70year male presented to us with complaints of dysuria. There was no history of flank pain. He was a known asthmatic and had undergone transurethral resection of prostate 7years back. Physical examination was unremarkable. Urinalysis showed $>100$ pus cells per high power field. Urine was sent for culture. Routine biochemical parameters were normal. Ultrasonography showed a calculus at the right ureterovesical junction. An unenhanced helical CT scan was done which showed a right ureterovesical calculus of size $10 \mathrm{~mm}$ and density $450 \mathrm{HU}$ (Figure 1). There was no hydronephrosis, hydroureter, perinephric or periureteral stranding. The CT was repeated in the prone position to differentiate whether it was calculus at the ureterovesical junction or if it was calculus in the bladder lying near the ureterovesical junction. The prone scan showed no change in the position of the calculus (Figure 2), which led to the conclusion that it was an ureterovesical junction calculus. Ureterorenoscopy was planned. However, on cystoscopy it was found that the calculus was not at the ureterovesical junction but was in a paraureteral diverticulum having a narrow mouth. It was dealt with by fragmentation using pneumatic lithotripsy and then removing the fragments.

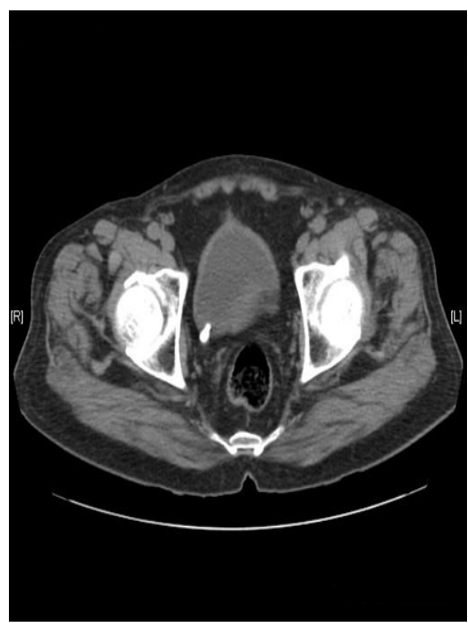

Figure I Unenhanced helical CT scan in supine position showing the calculus.

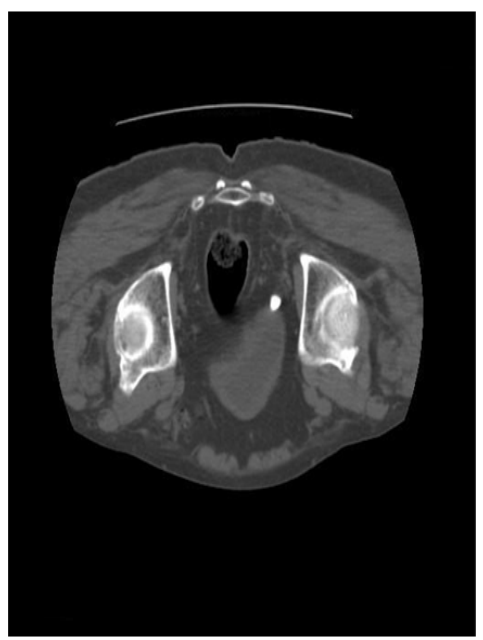

Figure 2 Unenhanced helical CT scan in Prone position. 


\section{Discussion}

Unenhanced Helical CT Scan is now an established modality to evaluate patients with renal colic. When a calculus is seen near the ureterovesical junction it is important to determine whether it is impacted at the ureterovesical junction or if it has already passed in the bladder and is lying near the ureterovesical junction. Clinical signs and symptoms cannot accurately differentiate between them. ${ }^{4}$ Ureteral dilatation and perinephric stranding are also not useful signs in differentiating between them. CT scan done in prone position helps to differentiate these two conditions. Calculus that does not change position is impacted at the ureterovesical junction while calculus which falls dependently in the prone position is in the bladder. ${ }^{3} \mathrm{We}$ had done a prone scan in our case to differentiate between the two. As there was no change in the position of the calculus, a diagnosis of calculus at the ureterovesical junction was made. On cystoscopy we found the calculus to be in a paraureteral diverticulum which had a narrow opening. This explained the findings of the prone CT scan. As the mouth of the diverticulum was narrow the calculus could not fall in a dependent position and the lack of change of position made us to diagnose it as being present at the ureterovesical junction.

It has been accepted that a prone $\mathrm{CT}$ scan will differentiate a stone impacted at the ureterovesical junction from a stone that has passed in the bladder. ${ }^{3}$ But, as there is an exception to every rule, similarly there is an exception to this finding. A calculus in a paraureteral diverticulum can mimic as a calculus impacted at the ureterovesical junction even in a prone position. We present this case to highlight this finding of a vesical calculus, in a paraureteral diverticulum, masquerading as a calculus at the ureterovesical junction on unenhanced helical CT scan.

\section{Acknowledgements}

None.

\section{Conflict of interest}

Author declares that there is no conflict of interest.

\section{References}

1. Smith RC, Verga M, Mc Carthy S, et al. Diagnosis of Acute Flank Pain: Value of unenhanced Helical CT. AJR. 1996;66(1):97-10.

2. Dalrymple NC, Verga M, Anderson K, et al. The value of unenhanced helical computerized tomography in the management of acute flank pain. J Urol. 1998;159(3):735-740.

3. Levine J, Neitlich J, Smith RC. The value of prone scanning to distinguish ueretrovesical junction stones from ureteral stone sthat have passed into the bladder: Leave No Stone Unturned. AJR. 1999;172(4):977-981.

4. Singal RK, Denstedt JD. Contemporary management of ureteral stones. Urol Clin North Am. 1997;24:59-68. 